\title{
Effects of tension on vortex-induced vibration (VIV) responses of a long tensioned cylinder in uniform flows
}

\author{
Ling Kang $^{1} \cdot$ Fei Ge $^{1} \cdot$ Xiaodong Wu${ }^{1} \cdot$ Youshi Hong ${ }^{1}$
}

Received: 14 December 2015 / Revised: 26 May 2016 / Accepted: 1 July 2016 / Published online: 29 November 2016

(C) The Chinese Society of Theoretical and Applied Mechanics; Institute of Mechanics, Chinese Academy of Sciences and Springer-Verlag Berlin Heidelberg 2016

\begin{abstract}
The effects of tension on vortex-induced vibration (VIV) responses for a tension-dominated long cylinder with an aspect ratio of 550 in uniform flows are experimentally investigated in this paper. The results show that elevated tension suppresses fluctuations of maximum displacement with respect to flow velocity and makes chaotic VIV more likely to appear. With respect to periodic VIV, if elevated tension is applied, the dominant vibration frequency in the in-line (IL) direction will switch from a fundamental vibration frequency to twice the value of the fundamental vibration frequency, which results in a ratio of the dominant vibration frequency in the IL direction to that in the cross-flow direction of 2.0. The suppression of the elevated tension in the fluctuation of the maximum displacement causes the axial tension to become an active control parameter for the VIV maximum displacement of a tension-dominated long riser or tether of an engineering structure in deep oceans. However, the axial tension must be optimized before being used since the high dominant vibration frequency due to the elevated tension may unfavorably affect the fatigue life of the riser or tether
\end{abstract}

Keywords Tension effect - Maximum displacement . Dominant vibration frequency $\cdot$ Chaos

Fei Ge

gefei@imech.ac.cn

$凶$ Youshi Hong

hongys@imech.ac.cn

1 Institute of Mechanics, Chinese Academy of Sciences, No. 15 Beisihuan Xi Road, Beijing 100190, China

\section{Introduction}

In the past two decades, a number of research efforts have focused on the behavior of the vortex-induced vibration (VIV) of elastically mounted rigid cylinders with one or two degrees of freedom. Comprehensive reviews on this topic for body-flow interaction are available in the literature, for example, Refs. [1-4]. Recent studies have been devoted to understanding the vibration behaviors of long flexible cylinders undergoing VIV in water. This is stimulated by the development of engineering structures in deep oceans, such as deep sea platforms and submerged floating tunnels (SFTs) [5]. Such constructions are aimed at meeting the needs of oil and gas exploration and public transportation in the deep ocean.

As important components of deep ocean structures, cylindrically shaped long risers and mooring tethers will behave differently from elastically mounted rigid bodies when subjected to VIV. Their dynamic responses are characterized by multiple and high modes, high harmonics, and traveling waves in addition to standing waves [6]. Dual-resonant and nonresonant responses are also concerned [7]. The effects of the flow profiles of sheared and uniform flows [8], Reynolds number [9], mass ratio [10], and suppression devices [11,12] on the VIV responses of long, flexible cylinders have been investigated experimentally. However, the effect of tension applied in the axial direction of long, flexible cylinders to prevent large deflections in the in-line (IL) direction of the cylinders due to drag force is less understood. Sanaati and Kato [13] pointed out that axial tension might significantly affect the order of the excited harmonics in the cross-flow (CF) direction and the IL direction. Thus axial tension seems to play an important role in the dynamic behavior of risers or mooring tethers and is considered an efficient method for the active control of VIV. 
Lee and Allen [14] noted that tensioned slender structures are classified into two types-beamlike (stiffnessdominated) and stringlike (tension-dominated) — by a dimensionless parameter, $T L^{2} / E I$, where $T$ is the tension, $L$ is the structure length, and $E I$ is the bending stiffness. If $T L^{2}$ is much greater than $E I$, then the structure is stringlike; otherwise, it is beamlike.

Gu et al. [15] investigated a model with an aspect ratio of 74.4 , a mass ratio of 1.55 , and a Reynolds number between 1950 and 19100 in stepped uniform flows. Six tensions were applied in the test, and the authors commented that the model was more like a beam rather than a string, where the bending stiffness played a more important role in the dynamic response of the model. We found that the values of $T L^{2} / E I$ corresponding to those tensions were 2.0, 3.0, 5.0, 7.1, 9.1, and 11.1

Huera-Huarte and Bearman [16] studied another model with an aspect ratio of 94 , a mass ratio of 1.80 , and a Reynolds number in the interval [1200, 12000] in stepped uniform flows. In the experiment, five different tensions were imposed. The authors concluded that as the tension increased, the model started to behave like a tension-dominated structure. The values of $T L^{2} / E I$ corresponding to those tensions were 5.6, 13.0, 22.3, 31.7, and 41.0.

Sanaati and Kato [13] used a model with an aspect ratio of 200, a mass ratio of 1.0, and a Reynolds number between 900 and 14400 in uniform flows. The model experienced four different tensions. The corresponding $T L^{2} / E I$ values were $80,147,267$, and 347 . The authors commented that the model was tension-dominated. In their experiments, higher tension resulted in a smaller CF displacement amplitude and generated a narrower lock-in bandwidth.

The comments of the authors in the previously mentioned three papers allow us to infer that if the dimensionless parameter $T L^{2} / E I$ is larger than 30 , the tensioned long and flexible cylinder would become a tension-dominated structure. Because the VIV responses of the models in the three aforementioned papers are similar to those of elastically mounted rigid bodies with one or two degrees of freedom, it is necessary to further investigate the effect of tension on tension-dominated long and flexible cylinders. The effects of tension on the characteristic responses of tension-dominated long and flexible cylinders are more complicated.

In this paper, a model with an aspect ratio of 550, a mass ratio of 1.50 , and a Reynolds number in the interval [600, 9000] was experimentally studied to explore the effect of axial tension on the VIV responses of tension-dominated long and flexible cylinders in uniform flows. The tension values are 10,50 , and $75 \mathrm{~N}$. The corresponding $T L^{2} / E I$ values are $30.4,152.2$, and 228.3. Obviously, the experimental model is tension-dominated. Research results show that the fluctuation in the maximum displacement with respect to the flow velocity is suppressed and the high dominant vibration fre- quency is excited as the tension increases. Thus, imposing a large axial tension is an efficient method for the active control of the VIV maximum displacement of a tension-dominated long riser or tether. However, a high dominant vibration frequency due to the large tension may unfavorably affect the fatigue life of the riser or tether. Therefore, the axial tension must be optimized when it is used as an active control parameter for the VIV maximum displacement of a tensiondominated long riser or tether.

\section{Experimental setup}

The experiment was carried out in a towing flume with a length of $29.0 \mathrm{~m}$, a width of $4.5 \mathrm{~m}$, and a depth of $4.0 \mathrm{~m}$ (Fig. 1a). As shown in Fig. 1b, the tested model was mounted on a towing car by universal joints. With such joints, the tested model has zero displacement and bending moments at both ends in all directions. The state of uniform flows was attained by having the towing car move forward or backward in the flume at a controllable and constant speed. One end of the tested model was connected by a cable, and using pulleys, the cable was hung with a steel weight inside a weight sleeve. With this setup, the tested model was tensioned.

An aluminum pipe with a circular cross section was used as the experimental model. Its length was $3.31 \mathrm{~m}$, its outer diameter was $6 \mathrm{~mm}$, and its inner diameter was $4 \mathrm{~mm}$. For each axial tension, the uniform flow velocity was varied from

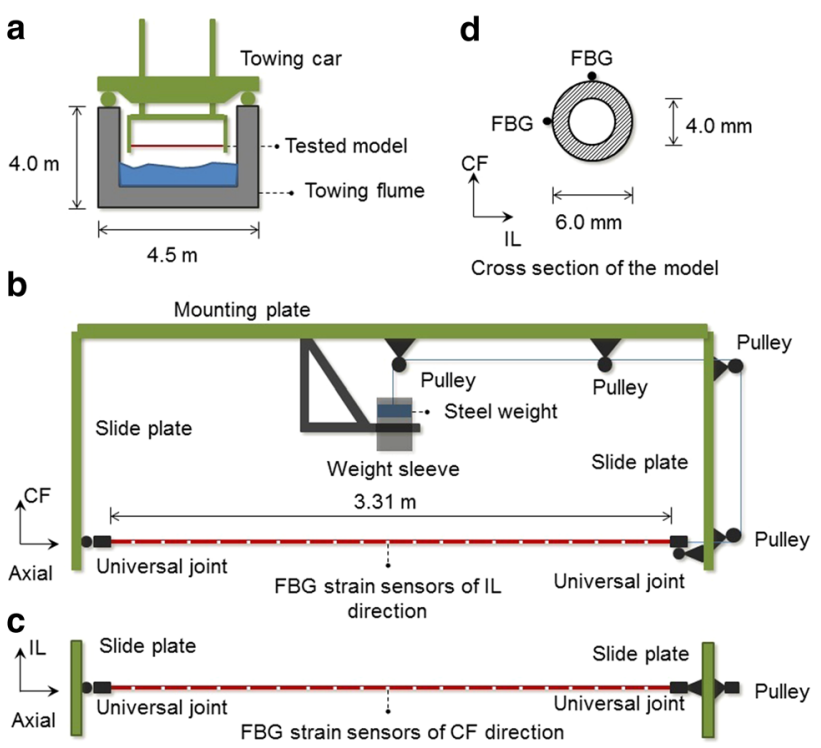

Fig. 1 Experimental setup. a Towing flume with tested model being mounted on a towing car. b Front view of experimental setup and arrangement of fiber brag grating (FBG) strain sensors in IL direction along axial direction of tested model. $\mathbf{c}$ Top view of experimental setup and arrangement of FBG strain sensors of CF direction along axial direction of tested model. d Cross section of tested model and arrangement of FBG strain sensors on cross section of tested model 
Table 1 Main experimental parameters

\begin{tabular}{llll}
\hline Parameter & Symbol & Unit & Value \\
\hline Length & $l$ & $\mathrm{~m}$ & 3.31 \\
Outer diameter & $d_{1}$ & $\mathrm{~mm}$ & 6.0 \\
Inner diameter & $d_{2}$ & $\mathrm{~mm}$ & 4.0 \\
Modulus of elasticity & $E$ & $\mathrm{GPa}$ & 71.0 \\
Tension & $T$ & $\mathrm{~N}$ & $10,50,75$ \\
Flow velocity & $U$ & $\mathrm{~m} / \mathrm{s}$ & $0.1-1.5$ \\
Aspect ratio & $l / d_{1}$ & - & 550 \\
Mass ratio & $m^{*}$ & - & 1.5 \\
Reynolds number & $R e$ & - & $600-9000$ \\
\hline
\end{tabular}

0.1 to $1.5 \mathrm{~m} / \mathrm{s}$, with an increment of $0.1 \mathrm{~m} / \mathrm{s}$. Table 1 summarizes the main parameters of the experiment. The mass ratio $m^{*}$ is the ratio of the model mass to the displaced water mass. The Reynolds number is $R e=U d_{1} / v$, where $v$ is the coefficient of the kinematic viscosity of water at $20^{\circ} \mathrm{C}$.

Fiber brag grating $(\mathrm{FBG})$ strain sensors stuck to the surface of the experimental model were used to measure the strains on the surface of the model. As illustrated in Fig. 1b-d, 20 FBG sensors were installed in the CF direction and another 20 in the IL direction with a sensor spacing of $160 \mathrm{~mm}$.

\section{Data analyses}

The maximum displacements along the axial direction of the model, the vibration frequencies, and the type of steady state of the vibration were derived from the measured strain signals.

\subsection{Displacement analysis}

The time series of the displacement of the model were extracted using the method in Refs. [8,17]. This method is based on the assumption that the displacement of a vibrating structure can be treated as the linear combination of a series of eigenfunctions of modes. By supposing that the eigenfunctions are sinusoidal or could be approximated as sinusoids, the vibration displacement $u(x, t)$ of the tested model takes the following form

$u(x, t)=\sum_{n=1}^{\infty} w_{1 n}(t) \sin \frac{n \pi}{l} x$,

where $w_{1 n}(t)$ is the $n$th modal weight of the displacement at time $t$.

According to the strain-displacement relationship of Euler-Bernoulli beams, the strain of the tested model is expressed as $\frac{\varepsilon(x, t)}{y}=\frac{\partial^{2} u(x, t)}{\partial x^{2}}=\sum_{n=1}^{\infty} w_{2 n}(t) \sin \frac{n \pi}{l} x$,

where $y$ is the distance from the neutral axis on the cross section of the tested model to the measured strain point. In this experiment, $y=d_{1} / 2 . w_{2 n}(t)$ is the $n$-th modal weight of the curvature at time $t$. The relationship between $w_{2 n}(t)$ and $w_{1 n}(t)$ is

$w_{2 n}(t)=-\left(\frac{n \pi}{l}\right)^{2} w_{1 n}(t)$.

We discretize Eqs. (1)-(3) in time and space and make the mode number finite. Then the discrete time series of displacement at each sensor can be calculated from the measured strain signals. Root mean squares (RMSs) of the time series of displacement at each sensor are then computed. The spatial maximum displacement is taken from the group of 20 RMS data in each direction. Because there may be a phase difference between the spatial maximum displacement in the $\mathrm{CF}$ direction and that in the IL direction, the total displacement is of great interest. Its time series was computed by

$u_{\mathrm{TOTAL}}(k)=\sqrt{u_{\mathrm{CF}}^{2}(k)+u_{\mathrm{IL}}^{2}(k)}$,

where $k$ is the discrete time of $k=0,1,2, \ldots$

Then the square percentages of the CF and the IL displacements in the total displacement at time $k$ are defined as

$p_{\mathrm{CF}}(k)=\frac{u_{\mathrm{CF}}^{2}(k)}{u_{\mathrm{TOTAL}}^{2}(k)} \times 100 \%=\frac{u_{\mathrm{CF}}^{2}(k)}{u_{\mathrm{CF}}^{2}(k)+u_{\mathrm{IL}}^{2}(k)} \times 100 \%$,

$p_{\mathrm{IL}}(k)=\frac{u_{\mathrm{IL}}^{2}(k)}{u_{\mathrm{TOTAL}}^{2}(k)} \times 100 \%=\frac{u_{\mathrm{IL}}^{2}(k)}{u_{\mathrm{CF}}^{2}(k)+u_{\mathrm{IL}}^{2}(k)} \times 100 \%$,

where $p_{\mathrm{CF}}(k)+p_{\mathrm{IL}}(k)=1$.

The values of the spatial maximum RMS of the total displacement are computed and the square percentages of the two vibration directions in the maximum mean squares (MS: square of the RMS) of the total displacement are given by $\left\langle\bar{p}_{\mathrm{CF}}\right\rangle$ and $\left\langle\bar{p}_{\mathrm{IL}}\right\rangle$, respectively. $\bar{p}_{\mathrm{CF}}$ and $\bar{p}_{\mathrm{IL}}$ are the temporal mean values of the time series defined by Eqs. (5) and (6) at each sensor location. $\left\langle\bar{p}_{\mathrm{CF}}\right\rangle$ and $\left\langle\bar{p}_{\mathrm{IL}}\right\rangle$ are the spatial mean values with respect to the sensor locations.

\subsection{Frequency and LLE analyses}

A fast Fourier transform (FFT) was applied to the discrete time series of displacement to obtain the frequency spec- 
trums. The spatial mean values of the frequency spectrums with respect to the sensor location were given.

The largest Lyapunov exponents (LLEs) were analyzed for the total displacement signals, of which the frequency spectrums were found to be wideband and random. This is because chaotic responses may occur in such situations. Lyapunov exponents are indices for describing the sensitivity to initial conditions in a dynamic system. The chaotic attractor of a dynamic system has wideband random frequency spectrums and is sensitive to initial conditions. Its two trajectories with nearby initial conditions will diverge, on average, at an exponential rate characterized by a positive LLE [18]. Therefore, a positive LLE is sufficient to diagnose chaos in a dynamic system. The method extracting LLE from a time series is based on the reconstruction of phase space. The algorithm used in this paper is described in Refs. [19,20]. Rosenstein et al. [19] proved that the algorithm employed to obtain LLE is reliable for small data sets, and it is fast and easy to implement.

\section{Results}

\subsection{Maximum displacements}

Figure 2 shows the results of the spatial maximum displacement. Column a shows the results for the case of $10 \mathrm{~N}$, whereas columns $\mathbf{b}$ and $\mathbf{c}$ are for the cases of 50 and $75 \mathrm{~N}$, respectively. The first row gives the spatial maximum RMS of the CF and IL displacements normalized by the outer diameter of the tested model, the second row illustrates the spatial maximum RMS of the total displacement normalized by the outer diameter, and the third row shows the square percentages of the two directions in the spatial maximum MS of the total displacement.

As shown in the first row, the maximum IL displacements have values close to the maximum CF displacement in most cases. When the tension increases, the maximum IL displacements in all tested flow velocity cases tend to drop to values no larger than the maximum CF displacement; in addition, with
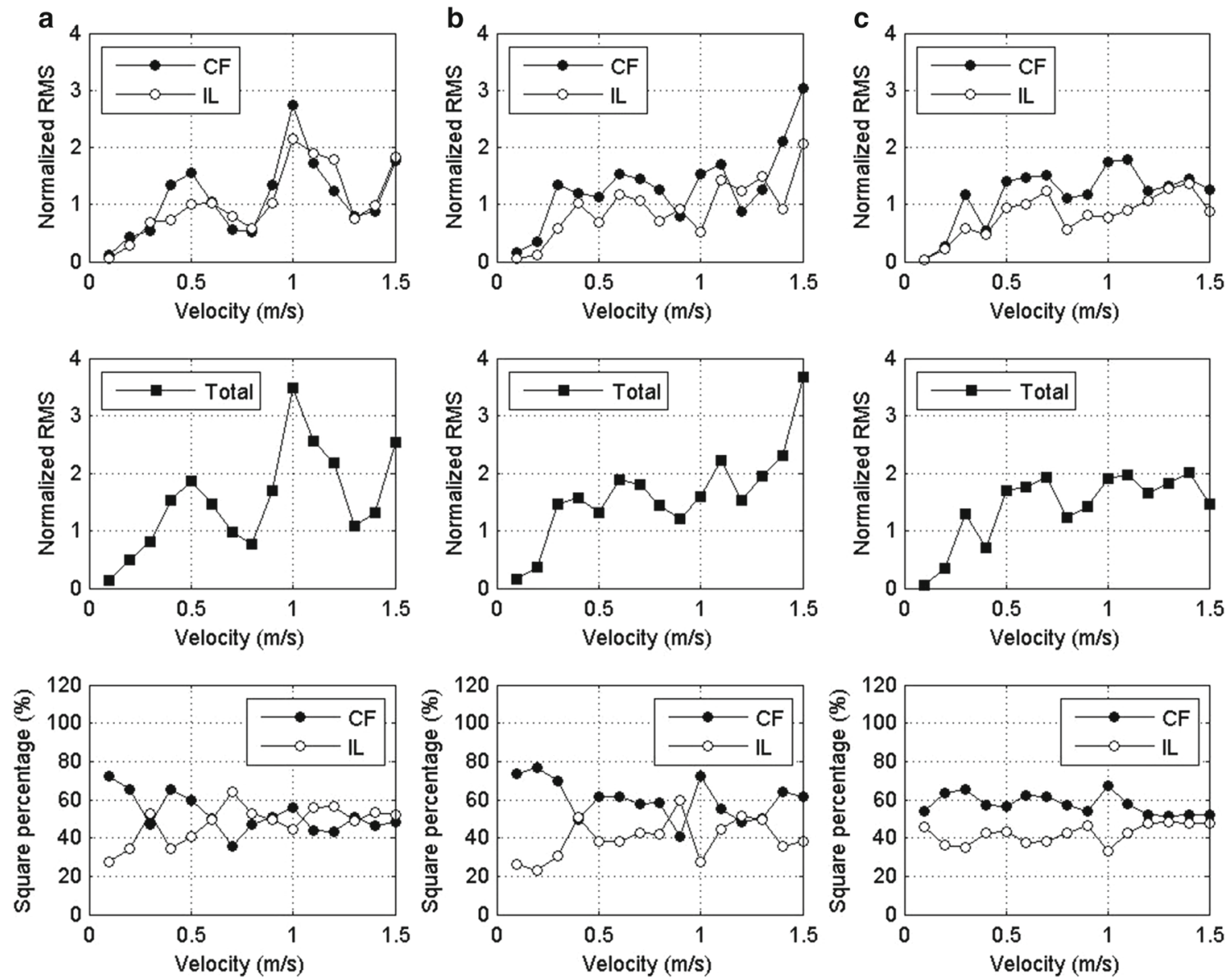

Fig. 2 First two rows spatial maximum RMS of displacement versus velocity. Third row: square percentages of spatial maximum MS of total displacement versus velocity for two directions. Tension in column: a $10 \mathrm{~N}, \mathbf{b} 50 \mathrm{~N}, \mathbf{c} 75 \mathrm{~N}$ 
Table 2 Mean square percentages with respect to flow velocity

\begin{tabular}{llll}
\hline Mean square (\%) & $10 \mathrm{~N}$ & $50 \mathrm{~N}$ & $75 \mathrm{~N}$ \\
\hline $\mathrm{CF}$ & 52.2 & 60.1 & 57.6 \\
$\mathrm{IL}$ & 47.8 & 39.9 & 42.4 \\
\hline
\end{tabular}

tension increases, the fluctuation of curves showing the relationship between the maximum displacement and the flow velocity in both CF and IL directions is suppressed. The maximum total displacement (second row) seems to show a trend that is similar to the maximum $\mathrm{CF}$ displacement when the flow velocity increases. The greater tension also results in a curve with relatively small fluctuations. The square percentage in the CF direction is always higher than that in the IL direction as the tension increases. Small changes in the square percentage with the flow velocity in both the CF and IL directions for higher tensions are present in the third row of Fig. 2. By averaging the square percentages with respect to the flow velocity, the mean values of the square percentage fluctuating with the flow velocity are obtained. As shown in Table 2, the square percentage in the CF direction is higher in the elevated tension cases (50 and $75 \mathrm{~N}$, of which the dimensionless parameters $T L^{2} / E I$ are one order of magnitude greater than that of $10 \mathrm{~N}$ ), which implies that the square percentage in the IL direction is lower in the same cases. That is to say, from a probabilistic point of view, the percentage of the CF displacement in the maximum total displacement is higher if greater tensions are imposed, and the percentage of the IL displacement in the maximum total displacement is lower.

Note that the maximum RMS of the displacement in the $\mathrm{CF}$ and IL directions approximately reached a value of $3 d_{1}$ and $2 d_{1}$, respectively. These values were never obtained in existing VIV studies. It is suggested that such high values of the maximum RMS of the displacement resulted from the small value of $T L^{2} / E I$. As shown in Fig. 2, the values of $3 d_{1}$ and $2 d_{1}$ first emerge at a flow velocity of $1.0 \mathrm{~m} / \mathrm{s}$ in the case of $10 \mathrm{~N}\left(T L^{2} / E I=30.4\right)$. Then it appears at a flow velocity of $1.5 \mathrm{~m} / \mathrm{s}$ in the case of $50 \mathrm{~N}\left(T L^{2} / E I=152.2\right)$. Finally, it disappears in the case of $75 \mathrm{~N}\left(T L^{2} / E I=228.3\right)$. This implies that large $T L^{2} / E I$ may suppress the value of the maximum RMS of the displacement in both the CF and IL directions.

\subsection{Vibration types}

Spectrums are categorized into three types based on the spatial mean frequency spectrums of the total displacement signals. Figure 3 presents examples of these types; the figure shows the results in the case of $10 \mathrm{~N}$. The flow velocities in Fig. 3a-c are $1.4,0.1$, and $0.7 \mathrm{~m} / \mathrm{s}$, respectively. As shown in
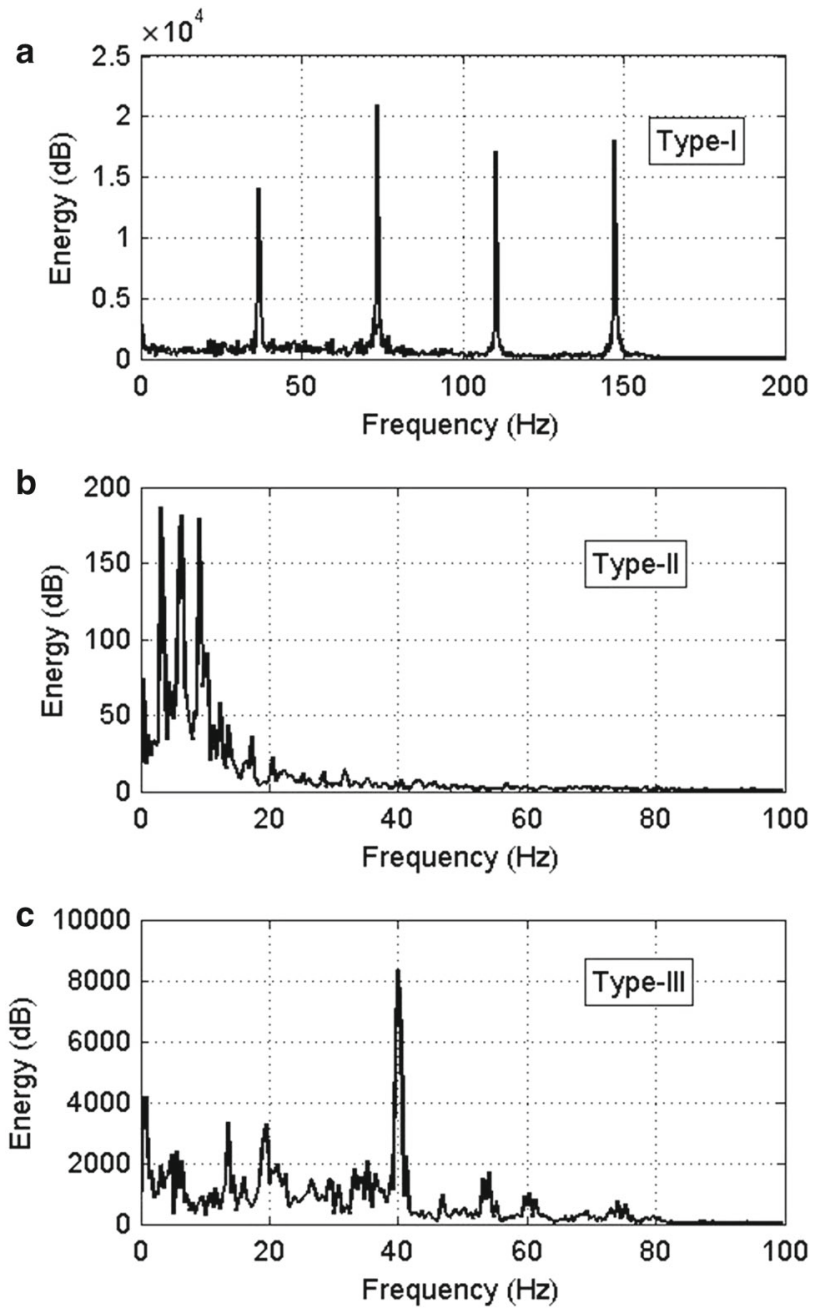

Fig. 3 Examples of three types of frequency spectrum found in the time series of total displacement. Flow velocity: a $1.4 \mathrm{~m} / \mathrm{s}, \mathbf{b} 0.1 \mathrm{~m} / \mathrm{s}$, c $0.7 \mathrm{~m} / \mathrm{s}$. Tested tension: $10 \mathrm{~N}$

Fig. 3a, Type I is the frequency spectrum that only includes several isolated peaks, indicating that the VIV response of the tested model is periodic and features multiple frequencies. Type II is a wideband spectrum with several peaks where the corresponding frequencies are multiples of one another. As shown in Fig. 3b, the first peak frequency is $3.2 \mathrm{~Hz}$, whereas the second one is $6.4 \mathrm{~Hz}$, that is, twice the first one, and the third is $9.2 \mathrm{~Hz}$, that is, approximately three times the first one. Type III is another kind of wideband spectrum, in which many peaks prevail, and they seem to be randomly distributed, as in Fig. 3c.

It is speculated that a chaotic response may emerge in Type III cases, so the LLE is calculated for those cases; the results are presented in Table 3, which shows that all LLEs are positive. Thus, it is concluded that the VIV responses with the frequency spectrum of Type III are all chaotic. Figure 4 displays the types of frequency spectrums of the total displacement for all cases. In the case of $10 \mathrm{~N}$, a chaotic 
Table 3 LLEs from total displacement signals with Type III spectrum

\begin{tabular}{|c|c|c|c|c|c|c|c|}
\hline \multirow[t]{2}{*}{ Case } & \multirow{2}{*}{$\begin{array}{l}10 \mathrm{~N} \\
0.7 \mathrm{~m} / \mathrm{s}\end{array}$} & \multicolumn{3}{|l|}{$50 \mathrm{~N}$} & \multicolumn{3}{|l|}{$75 \mathrm{~N}$} \\
\hline & & $0.1 \mathrm{~m} / \mathrm{s}$ & $0.2 \mathrm{~m} / \mathrm{s}$ & $0.8 \mathrm{~m} / \mathrm{s}$ & $0.1 \mathrm{~m} / \mathrm{s}$ & $0.2 \mathrm{~m} / \mathrm{s}$ & $0.4 \mathrm{~m} / \mathrm{s}$ \\
\hline LLE & 47.4 & 24.4 & 22.0 & 34.6 & 62.8 & 21.1 & 24.3 \\
\hline
\end{tabular}

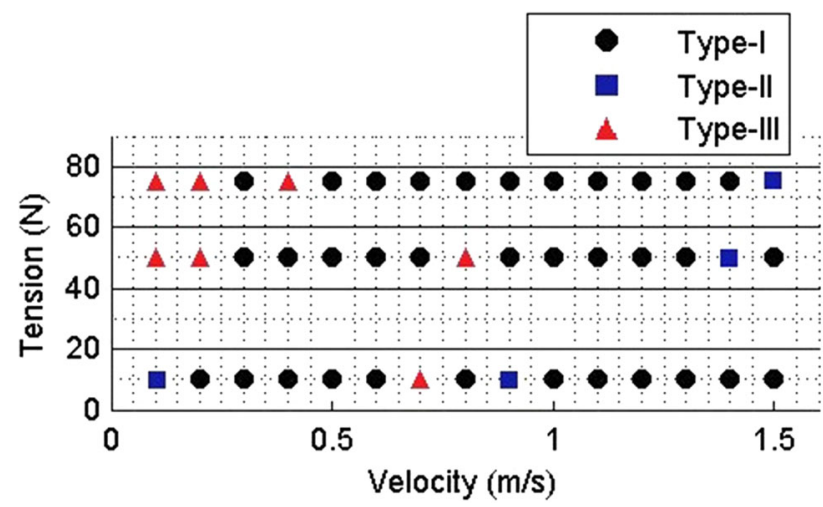

Fig. 4 Types of spatial mean frequency spectrum found in time series of total displacement for all tested cases

response appears only in one flow velocity case, while in the cases of 50 and $75 \mathrm{~N}$, such a response is observed in three flow velocity cases. It is concluded that in cases with elevated tension, a chaotic response appears in more flow velocity cases, implying that a chaotic response is more likely to appear in cases of the elevated tension.

\subsection{Vibration frequencies}

The peak frequencies of the periodic responses and those with Type II spatial mean frequency spectrum are plotted in Figs. 5-7, which respectively show the results in the CF, IL, and total directions. In Figs. 5-7, panel a presents the results of the case of $10 \mathrm{~N}$; panels $\mathbf{b}$ and $\mathbf{c}$ illustrate the results of the cases of 50 and $75 \mathrm{~N}$, respectively. Note that the smallest peak frequency in the CF direction here is defined as the fundamental vibration frequency, symbolized by $x$.

As indicated by Fig. 5, the dominant vibration frequency (the energy is the largest) in the CF direction is generally $1 \times$ in all tension cases. A frequency of $2 \times$ also prevails in the CF direction and seems to be dominant at high flow velocities. A frequency of $3 \times$ is observed at some flow velocities in the CF direction. However, the energy of a frequency of $3 \times$ is obviously the smallest in the spectrum. Tension does not disrupt this kind of trend. In the IL direction, as shown in Fig. 6, the dominant vibration frequency is likely to switch from $1 \times$ to $2 \times$ as the tension increases. That is to say, for the elevated tension cases, a frequency of $2 \times$ becomes dominant and more important. Thus the ratio of the IL dominant vibration frequency to the $\mathrm{CF}$ dominant vibration frequency equals 2.0.

In practice, the vibration behaviors of the tested model will agree with those shown by the total displacement, not just those coming from CF and IL components. Therefore, peak frequencies in the total displacement signals are also shown. As illustrated in Fig. 7, the frequency spectrum from the total displacement signal is more abundant than that from just the CF component and the IL component. In addition
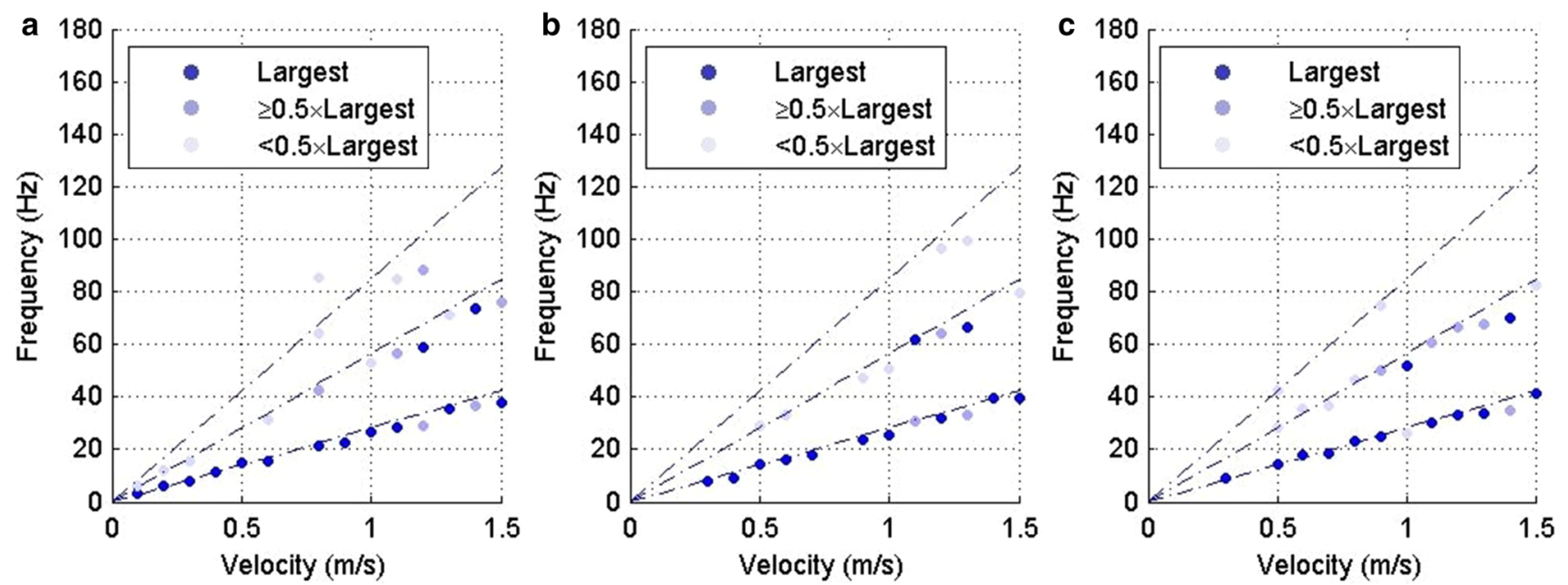

Fig. 5 (Color online) Peak frequencies in CF direction with Type I and Type II spatial mean frequency spectrum: a 10 N, b 50 N, c 75 N. Solid circles, which are in the deepest color, indicate that the energy of the corresponding frequency is the largest in the spectrum, whereas the second deepest color indicates that the energy of the corresponding frequency is no less than $50 \%$ of the largest, and the lightest one is the case where the energy of the corresponding frequency is less than $50 \%$ of the largest. Dash-dotted lines represent the Strouhal frequency $\left(f_{\mathrm{st}}\right)$ and its multiples $\left(2 f_{\text {st }}, 3 f_{\text {st }}\right)$ versus flow velocity, where the Strouhal number was considered to be 0.17 

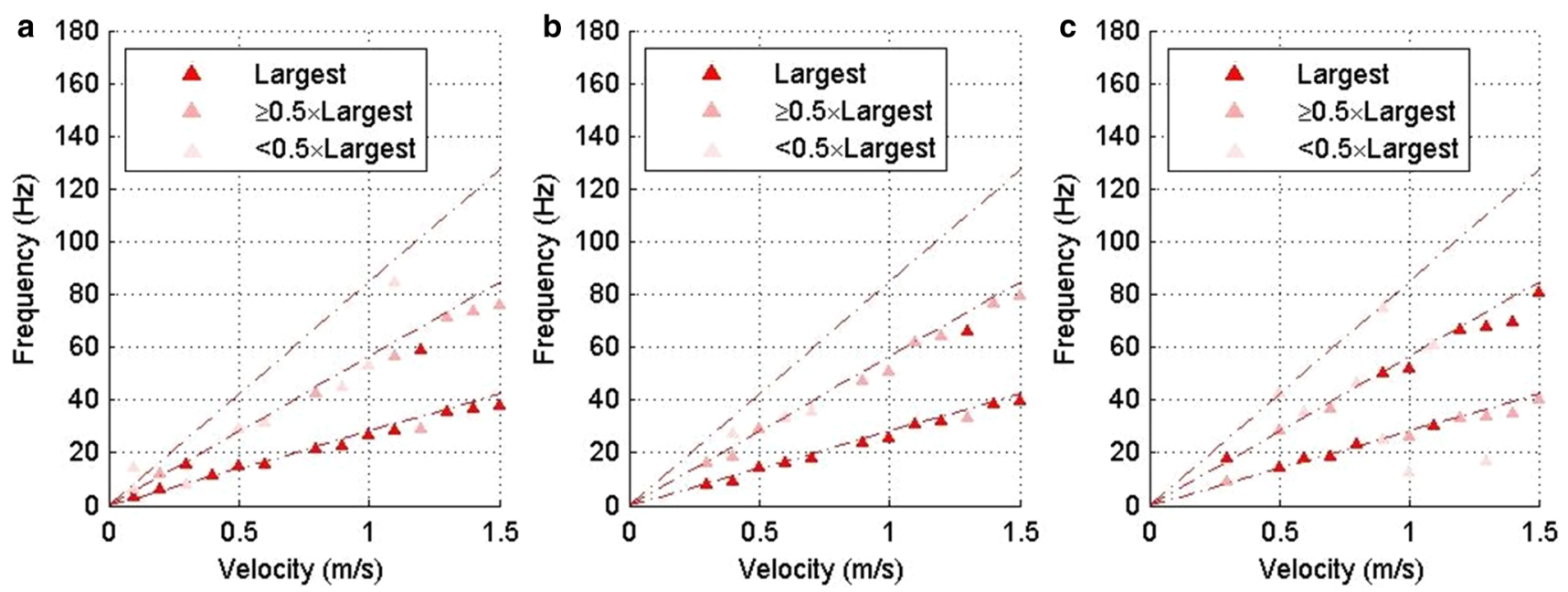

Fig. 6 (Color online) Peak frequencies in IL direction with Type I and Type II spatial mean frequency spectrum: a 10 N, b 50 N, c 75 N. Solid triangles, which are in the deepest color, indicate that the energy of the corresponding frequency is the largest in the spectrum, whereas the second deepest color indicates that the energy of the corresponding frequency is no less than $50 \%$ of the largest, and the lightest one is the case where the energy of the corresponding frequency is less than $50 \%$ of the largest. Dash-dotted lines represent the Strouhal frequency $\left(f_{\mathrm{st}}\right)$ and its multiples $\left(2 f_{\text {st }}, 3 f_{\text {st }}\right)$ versus flow velocity, where the Strouhal number was considered to be 0.17
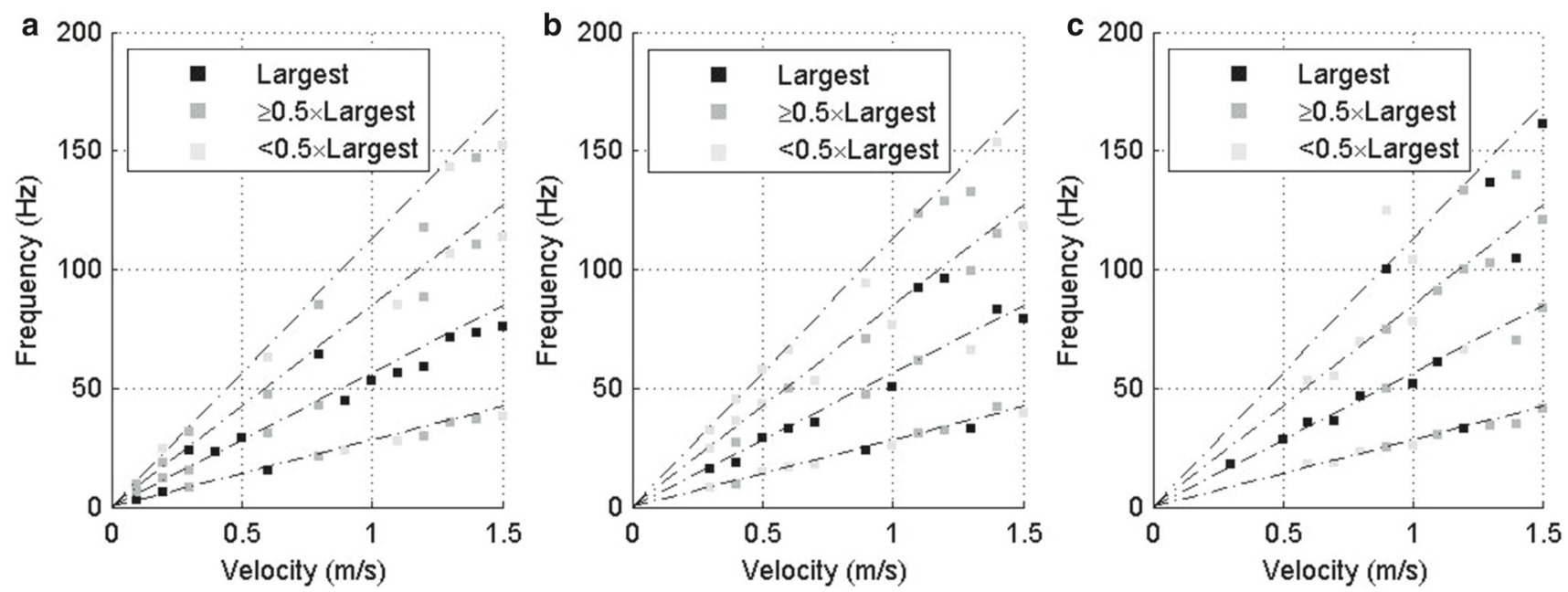

Fig. 7 (Color online) Peak frequencies of total displacement signals with Type I and Type II spatial mean frequency spectrum: a $10 \mathrm{~N}$, b $50 \mathrm{~N}$, c 75 N. Solid squares, which are in the deepest color, indicate that the energy of the corresponding frequency is the largest in the spectrum, whereas the second deepest color indicates that the energy of the corresponding frequency is no less than $50 \%$ of the largest, and the lightest one denotes the case where the energy of the corresponding frequency is less than $50 \%$ of the largest. Dash-dotted lines represent the Strouhal frequency ( $\left.f_{\text {st }}\right)$ and its multiples $\left(2 f_{\mathrm{st}}, 3 f_{\mathrm{st}}, 4 f_{\mathrm{st}}\right)$ versus flow velocity, where the Strouhal number was considered to be 0.17

to frequencies of $1 \times$ and $2 \times$, frequencies of $3 \times$ and $4 \times$ appear in the total spectrum. In the low-tension case $(10 \mathrm{~N})$, the dominant vibration frequency in the total spectrum scatters at frequencies of $1 \times, 2 \times$, and $3 \times$ within the low flow velocity region from 0.1 to $0.8 \mathrm{~m} / \mathrm{s}$, while the distribution converges at a frequency of $2 \times$ within the high flow velocity region from 0.9 to $1.5 \mathrm{~m} / \mathrm{s}$. Conversely, for high-tension cases $(50 \mathrm{~N}, 75 \mathrm{~N})$, the dominant vibration frequency converges at a frequency of $2 \times$ within the low velocity region, whereas within the high flow velocity region, it scatters. For the case of $50 \mathrm{~N}$, it scatters at frequencies of $1 \times, 2 \times$, and $3 \times$. For the case of $75 \mathrm{~N}$, it mainly scatters at frequencies of $2 \times$,
$3 \times$, and $4 \times$, and the dominant vibration frequency is more likely to jump to a frequency of $4 \times$. This implies that if elevated tension is applied, it is much easier for the large peak frequencies in the total spectrum, such as $3 \times$ and $4 \times$ in this paper, to become dominant vibration frequencies at high flow velocities.

As described earlier, the dominant vibration frequency is a multiple of the fundamental vibration frequency. Therefore, the fundamental frequency is a quantity that is essential for identifying the dominant vibration frequency. Thus, it is of great interest in which parameters determine the fundamental vibration frequency. 

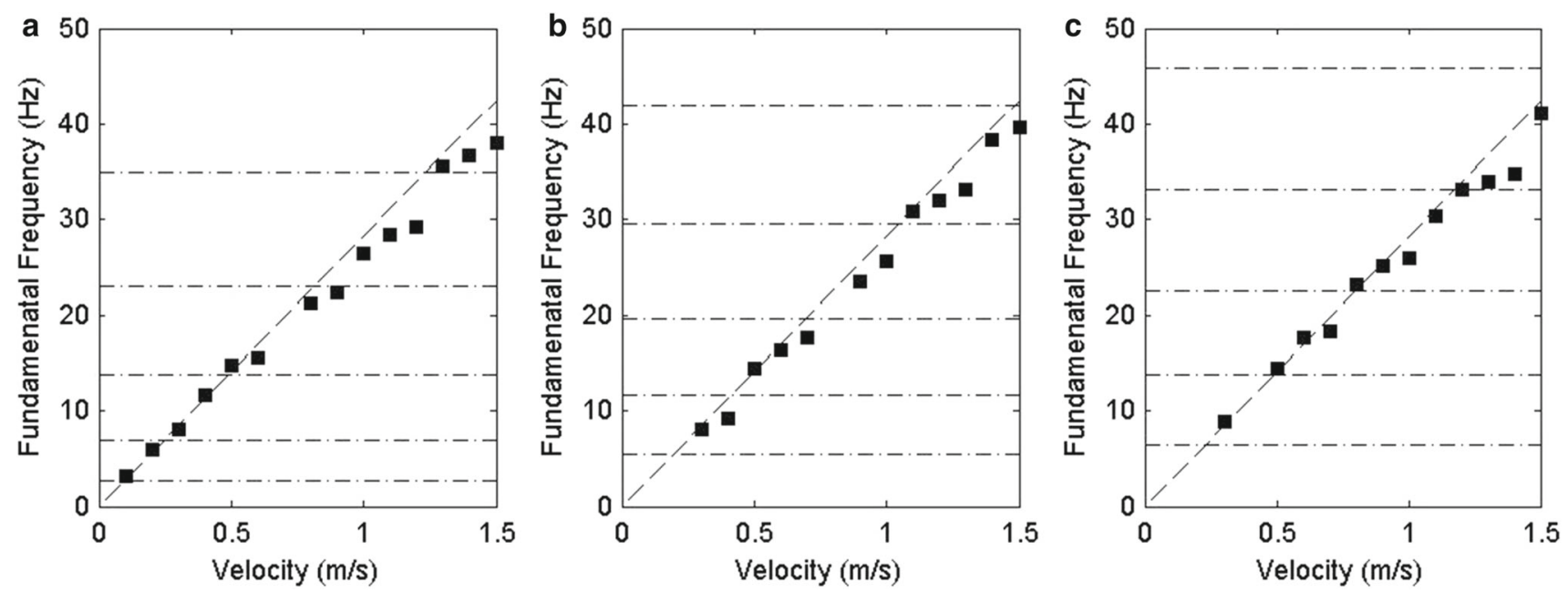

Fig. 8 Fundamental frequencies with Type I and Type II spatial mean frequency spectrum versus flow velocity: a $10 \mathrm{~N}$, b $50 \mathrm{~N}$, c $75 \mathrm{~N}$. The dashed line represents the Strouhal frequency $\left(f_{\mathrm{st}}\right)$ versus flow velocity, where the Strouhal number was taken as 0.17. Dash-dotted lines represent the natural frequency lines. Bottom to top: orders of natural frequencies are 1-5

Figure 8 shows the fundamental vibration frequency versus flow velocity for all tension cases. As indicated, the fundamental vibration frequencies are not completely in accordance with the Strouhal frequencies (the Strouhal number is 0.17). In several cases, they are deviated from the Strouhal frequency values and have a stepped increase as the flow velocity increases, which is similar to the case given in Ref. [21]. It is also observed that the fundamental vibration frequencies are affected by the natural frequencies of the tested model and, thus, by tension. The natural frequencies are computed using the following equation:

$f_{n}=\frac{n^{2} \pi}{2 l^{2}} \sqrt{\frac{E I}{m}\left(1+\frac{T L^{2}}{n^{2} \pi^{2} E I}\right)}$,

where the order $n=1,2, \ldots$, and the mass $m$ is the sum of the structural mass and the added mass (an added mass coefficient of 1.0 is applied).

According to Eq. (7), when the tension increases, the natural frequencies increase. Thus, the intersection points between the natural frequency lines, and the Strouhal frequency line moves in the direction of increasing flow velocity. The fundamental vibration frequencies, as illustrated in Fig. 8, change their values in the upper right shift of the intersection points. Take a flow velocity of $0.6 \mathrm{~m} / \mathrm{s}$ as an example. In the case with the tension being $10 \mathrm{~N}$, the fundamental vibration frequency is $15.6 \mathrm{~Hz}$, which is between the Strouhal frequency of $17.0 \mathrm{~Hz}$ and the third natural frequency of $13.8 \mathrm{~Hz}$. However, in the tension case of $50 \mathrm{~N}$, with the third natural frequency being $19.6 \mathrm{~Hz}$, and the tension case of $75 \mathrm{~N}$, with the third natural frequency being $22.5 \mathrm{~Hz}$, the fundamental vibration frequency becomes 16.4 and $17.6 \mathrm{~Hz}$, respectively. These fundamental vibration frequencies are more likely to agree with a Strouhal frequency of $17.0 \mathrm{~Hz}$, where the third natural frequency is higher but the second natural frequency is not high enough to affect the fundamental vibration frequency. A similar situation is found at a flow velocity of $1.4 \mathrm{~m} / \mathrm{s}$. In this case, the point where the fifth natural frequency line intersects the Strouhal frequency line moves to the upper right as the tension increases. The only difference is that for the case of $75 \mathrm{~N}$, the fourth natural frequency is high enough to affect the fundamental vibration frequency and causes it to decrease to $34.8 \mathrm{~Hz}$, which is close to the fourth natural frequency, $33.1 \mathrm{~Hz}$.

\section{Conclusions}

The effects of tension on the VIV responses of a tensiondominated long and flexible cylinder in uniform flows were experimentally investigated. The following conclusions are drawn.

As the tension increases, the maximum IL displacement tends to drop to values no larger than the maximum CF displacement at all tested flow velocities. Fluctuations in the maximum displacement with respect to the flow velocity in both the CF and IL directions diminish. The maximum total displacement follows the trend of the maximum CF displacement when the flow velocity changes. For high-tension cases, the percentage of IL displacement in the maximum total displacement is reduced, implied that IL displacement is less important than $\mathrm{CF}$ displacement when high tension is imposed.

Three types of frequency spectrum are found in all total displacement signals: (1) spectrum with several isolated peaks (Type I), (2) wideband spectrum with several peaks that are multiples of one another (Type II), and (3) wide- 
band spectrum with random distributed peaks (Type III). It is quantitatively proved by the LLE that the vibration response with the Type III frequency spectrum is chaotic. High tension seems to make it much easier for a chaotic response to emerge.

With respect to the periodic responses and those with a Type II frequency spectrum, the effect of tension on the dominant vibration frequency in the $\mathrm{CF}$ direction is not significant, whereas high tension may switch the dominant vibration frequency in the IL direction from a fundamental vibration frequency to a frequency that is twice the fundamental vibration frequency. As a result, the ratio of the IL dominant vibration frequency to the $\mathrm{CF}$ dominant vibration frequency becomes 2.0. The higher peak frequencies existing in the total spectrum probably become the dominant vibration frequencies at high flow velocities if the elevated tension is applied. The fundamental vibration frequencies are affected not only by the flow velocity but also by the tension.

Acknowledgements This project was supported by the Construction Technology Program of the Ministry of Transport (Grant 2013318 740050).

\section{References}

1. Bearman, P.W.: Circular cable wakes and vortex-induced vibrations. J. Fluid Struct. 27, 648-658 (2011)

2. Gabbai, R.D., Benaroya, H.: An overview of modeling and experiments of vortex induced vibration of circular cylinders. J. Sound Vib. 282, 575-616 (2005)

3. Sarpkaya, T.: A critical review of the intrinsic nature of vortexinduced vibrations. J. Fluid Struct. 19, 389-447 (2004)

4. Williamson, C.H.K., Govardhan, R.: Vortex-induced vibrations. Annu. Rev. Fluid Mech. 36, 413-455 (2004)

5. Ahrens, D.: Submerged floating tunnels-a concept whose time has arrived. Tunn. Undergr. Sp. Tech. 12, 317-336 (1997)

6. Wu, X.D., Ge, F., Hong, Y.S.: A review of recent studies on vortexinduced vibrations of long flexible cylinders. J. Fluid Struct. 28, 292-308 (2012)

7. Wu, X.D., Ge, F., Hong, Y.S.: An Experimental investigation of dual-resonant and non-resonant responses for vortex-induced vibration of a long slender cylinder. Sci. China Phys. Mech. 57, 321-329 (2014)
8. Trim, A.D., Braaten, H., Lie, H., et al.: Experimental investigation of vortex induced vibration of long marine risers. J. Fluid Struct. 21, 335-361 (2005)

9. Resvanis, T.L., Jhingran, V., Vandiver, J.K., et al.: Reynolds number effects on the vortex-induced vibration of flexible marine risers. In: Proceedings of the ASME 2012 31st International Conference on Ocean, Offshore and Arctic Engineering, Brazil, OMAE201283565 (2012)

10. Huera-Huarte, F.J., Bangash, Z.A., Gonzalez, L.M.: Towing tank experiments on the vortex-induced vibrations of low mass ratio long flexible cylinder. J. Fluid Struct. 48, 81-92 (2014)

11. Wu, H., Sun, D.P., Lu, L., et al.: Experimental investigation on the suppression of vortex-induced vibration of long flexible riser by multiple control rods. J. Fluid Struct. 30, 115-132 (2012)

12. Huera-Huarte, F.J.: On splitter plate coverage for suppression of vortex-induced vibrations of flexible cylinders. Appl. Ocean Res. 48, 244-249 (2014)

13. Sanaati, B., Kato, N.: Vortex-induced vibration (VIV) dynamics of a tensioned flexible cylinder subjected to uniform cross-flow. J. Mar. Sci. Technol. 18, 247-261 (2013)

14. Lee, L., Allen, D.: Vibration frequency and lock-in bandwidth of tensioned flexible cylinders experiencing vortex shedding. J. Fluid Struct. 26, 602-610 (2010)

15. Gu, J.J., Vitola, M., Coelho, J., et al.: An experimental investigation by towing tank on VIV of a long flexible cylinder for deepwater riser application. J. Mar. Sci. Technol. 18, 358-369 (2013)

16. Huera-Huarte, F.J., Bearman, P.W.: Wake structures and vortexinduced vibrations of a long flexible cylinder-Part I: dynamic response. J. Fluid Struct. 25, 969-990 (2009)

17. Lie, H., Kaasen, K.E.: Modal analysis of measurements from a large-scale VIV model test of a riser in linearly sheared flow. J. Fluid Struct. 22, 557-575 (2006)

18. Eckmann, J.-P., Ruelle, D.: Ergodic theory of chaos and strange attractors. Rev. Mod. Phys. 57, 617-656 (1985)

19. Rosenstein, M.T., Collins, J.J., De Luca, C.J.: A practical method for calculating largest Lyapunov exponents from small data. Phys. D 65, 117-134 (1993)

20. Wu, Y., Su, J., Tang, H., et al.: Analysis of the emergence in swarm model based on largest Lyapunov exponent. Math. Probl. Eng. 2011, 745257 (2011)

21. Chaplin, J.R., Bearman, P.W., Huera-Huarte, F.J., et al.: Laboratory measurements of vortex-induced vibrations of a vertical tension riser in a stepped current. J. Fluid Struct. 21, 3-24 (2005) 